\title{
Resultados de la implementación de registros médicos electrónicos (EMR) a nivel regional
}

\author{
Albarracín Ricardo ${ }^{1}$ \\ ${ }^{1}$ Universidad Central del Ecuador, Facultad de Ingeniería, Ciencias Físicas y Matemática, Instituto de \\ Investigación y Posgrado, Quito, Ecuador \\ e-mail: ralbarracin@ups.edu.ec \\ Información del artículo \\ Recibido: Junio 2015 - Aceptado: Agosto 2015
}

\begin{abstract}
Resumen
Los sistemas de información en salud (SIS) han sido implementados desde hace varias décadas en distintos países, no solo en los desarrollados, sino también en países de América Latina. En el Ecuador se está utilizando estos sistemas en pocos centros de salud y hospitales, principalmente por la complejidad y el alto costo que representa su desarrollo y mantenimiento. En la mayoría de hospitales y centros médicos de salud del país, será necesario invertir una cantidad considerable de recursos económicos para contar con la infraestructura tecnológica adecuada que permita implementar los sistemas de registros médicos electrónicos (EMR). Además de la falta de infraestructura en Tecnologías de la Información y Comunicación (TIC) en los hospitales, surgen otros problemas, sobre la calidad de la información, la estandarización y el riesgo que implica que la información sanitaria de los pacientes sea accedida por usuarios no autorizados. En este artículo se investiga los sistemas EMR implementados en otros países, especialmente de América Latina, identificando los casos de éxito y los principales beneficios de su aplicación. Finalmente, en el caso de Ecuador se presenta algunos datos del sistema "Si Salud" que ha sido implementado como plan piloto en algunas instituciones de salud, pertenecientes al Ministerio de Salud Pública (MSP), para terminar con una comparación entre las historias clínicas electrónicas (HCE) y las historias clínicas en papel, recalcando que para desarrollar un SIS es necesario contar con normativas y estándares a nivel nacional para lograr la interoperabilidad de los sistemas informáticos relacionados con el sistema de salud.
\end{abstract}

Palabras clave: sistemas de información en salud (SIS), historia clínica electrónica (HCE), servicios de salud, estándares para interoperabilidad de sistemas.

\begin{abstract}
Health Information Systems (HIS) had been implemented for decades in many countries, not only in developed countries but also in Latin America. Few health centers and hospitals are using this kind of systems in Ecuador, because of the complexity and the high cost that represents the development and maintenance of the system. The majority of hospitals and health centers of the country, know that it will be necessary to invest a big and considerable amount of economic resources to count with the right technologic infrastructure to implement electronic medical record systems. There are many problems to get a system of information besides the lack of infrastructure in Information Technology and Communication (ICT) in hospitals. These problems involve the quality of information, standardization and the risk of the patient health information would be accessed by unauthorized users. This paper investigates implemented electronic medical record (EMR) systems in other countries, especially in Latin America identifying the success cases and mainly benefits of its implementation. Finally, Ecuador's case presents some information of the system "Si Salud" which had been implemented as a pilot program in many health institutions belonging to the Ministry of Public Health (MHP). As a conclusion I want to make a comparison between electronic health record (HER), and health records that are organized and saved by paper files. It is very important and necessary to know the international normative and standards to get the interoperability of technician systems related to health.
\end{abstract}

Keywords: health information systems (HIS), electronic health record (HER), health services, standards of systems interoperability. 


\section{Introducción}

Los sistemas de información son utilizados en todos los ámbitos de la sociedad. En el campo de la salud los primeros sistemas en informatizarse fueron los que apoyaban los procesos administrativos, pero no mejoraron la gestión clínica significativamente. Actualmente se cuenta con sistemas de salud en todas sus áreas, siendo de vital importancia la información clínica que permite que los datos personales y clínicos de los pacientes y las acciones de los médicos se almacenen en los sistemas de información.

En el desarrollo de los sistemas de información en salud (SIS) aparecen las historias clínicas electrónicas (HCE), que tienen beneficios significativos en comparación con las historias clínicas manejadas en papel. Las HCE han sido adoptadas a nivel mundial, especialmente por la seguridad y disponibilidad en el manejo de la información de los pacientes, y además porque mejoran la toma de decisiones por parte de los profesionales de la salud y organismos de salud pública de cada país [1].

Los SIS almacenan la información contenida en la historia clínica de un paciente y permiten guardar datos adicionales como: resultados de laboratorio, diagnósticos, exámenes radiológicos, prescripción de medicamentos, tratamientos y resultados de esos tratamientos. Por esta razón la cantidad de información relacionada a sistemas de salud creció significativamente, siendo necesario contar con mecanismos y herramientas, que permitan almacenar el gran volumen de información, así como también contar con software especializado para realizar búsquedas rápidas y eficientes por parte de los profesionales de la salud.

El presente documento sintetiza el desarrollo de los sistemas de "Registros Médicos Electrónicos" (RME) en los Estados Unidos, Europa y algunos países de América Latina. Se presentan los casos de éxito y los mecanismos o estrategias que utilizaron para mejorar los servicios de salud. En cuanto a las historias clínicas electrónicas (HCE), se indica su definición, su estructura y las principales ventajas con respecto a las historias clínicas en papel. Finalmente se describen algunos estándares, protocolos de comunicación, formatos de intercambio de datos y herramientas utilizados para lograr la interoperabilidad entre los sistemas.

\section{Materiales y metodología}

Se realizó una investigación preliminar acerca de los sistemas de información de salud desarrollados en varios países, se revisó artículos académicos que trataban sobre las políticas, estrategias, componentes y estándares de un sistema de información en salud, que fueron utilizados para el desarrollo de estos sistemas en Estados Unidos, Europa y algunos países de Latinoamérica. Con la información recopilada se procedió a elaborar estrategias para analizar y seleccionar la información y contar con elementos que permitan comprobar la hipótesis. En la primera parte se identificó la idea predominante o mensaje principal que aborda el artículo. Con la idea principal se procedió a definir claramente qué se quiere que haga el documento, identificando el propósito a lograr y hacia cuáles lectores estaría enfocado el artículo. Para desarrollar el concepto fue necesario elaborar un relato, estructurando las ideas y reuniendo los datos que sustentan esas ideas, para finalmente escribir el esquema que muestra las ideas fundamentales y sus relaciones.

Para la generación de ideas se utilizó dos técnicas: los mapas mentales; y, la agrupación y síntesis. Los mapas mentales fueron utilizados para la primera etapa de reflexión, en cambio mediante la agrupación y síntesis se procedió a identificar los puntos fundamentales. Con estas dos técnicas se elaboró el esquema que permitió guiar la investigación y comprobar o rechazar la hipótesis.

Finalmente, con base en el esquema se realizó el diseño de la investigación, seleccionando múltiples fuentes de información, en las cuales se describía los sistemas de información en salud, las historias clínicas electrónicas, los estándares para la interoperabilidad de los sistemas y las diferentes categorías de estándares existentes para la transferencia de información entre los sistemas.

\section{A. Sistemas de registros médicos electrónicos, EMR}

Un nuevo sistema de información en la mayoría de los casos afecta a los procesos de negocio de una empresa, por lo que se debe analizar en qué medida afectará en el giro del negocio. Un nuevo sistema no solo es hardware y software, sino que involucra un cambio organizacional y administrativo en las empresas e incidirá en la forma como las personas realizan sus trabajos [2]. El desarrollo de los sistemas EMR es comple- 
jo, difícil de mantener a largo plazo y con costos relativamente altos.

\section{B. Antecedentes}

Los sistemas EMR han sido desarrollados por varios países del mundo, para mejorar los servicios de salud a sus pacientes, pero en la mayoría de los casos no han cumplido con las expectativas que tenían antes de su implementación. La falta de estándares ha sido una de las principales causas, para que al poco tiempo dejen de ser utilizados en centros de salud y hospitales. La salud de los habitantes de América Latina ha experimentado mejoras durante las últimas décadas. Sin embargo, hay algunas metas que todavía no se han alcanzado, hay grandes retos tanto con las enfermedades transmisibles como con las no transmisibles. Por ejemplo, ha habido un incremento en las enfermedades no transmisibles como la obesidad, la diabetes, las enfermedades cardiovasculares y el cáncer.

Los recursos humanos, la infraestructura insuficiente y las grandes diferencias geográficas y culturales aumentan la complejidad de la situación en la Región. La disponibilidad de servicios de salud y de información relacionada con la salud varía mucho entre comunidades, zonas geográficas y países, obstaculizando el acceso universal a servicios de salud y disminuyendo la calidad de la atención. Estas diferencias se deben a barreras geográficas, procesos y decisiones políticas. La difusión y adopción de las tecnologías de la información y comunicación, ofrecen la posibilidad de establecer condiciones de igualdad, al disminuir algunas de las barreras y propiciar un intercambio de información. La manera de lograr condiciones de igualdad y permitir el acceso universal a servicios de salud es mediante el aumento de la inversión en servicios de salud con respecto al Producto Interno Bruto (PIB). La figura 1 muestra las cifras de inversión del Ecuador en servicios de salud. Si bien las cifras de inversión se han incrementado año tras año, no se conoce estadísticas de los valores económicos invertidos en sistemas de información en salud y las mejoras en los servicios de salud producidos por estos incrementos.

La inversión del gobierno en el sistema de registros médicos electrónicos, implementado a inicios del 2011 en el Hospital de Especialidades Eugenio Espejo (HEEE) de la ciudad de Quito no se conoce, y tampoco las razones por las que al poco tiempo de haber sido instalado, se haya dejado de utilizarlo. Se terminó el contrato con la empresa desarrolladora y el hospital pasó a utilizar nuevamente las historias clínicas en papel hasta la actualidad, ocasionando demoras significativas en la atención de los pacientes en los servicios de salud.

El desarrollo de los sistemas de información en salud, en los países de la Región de las Américas está en sus inicios, son en su mayoría aislados y utilizan tecnologías obsoletas con funcionalidades limitadas. Al final los profesionales de la salud no pueden acceder a la información almacenada en estos sistemas, ni tampoco tomar decisiones acertadas y a tiempo.

Para evitar estos problemas, al desarrollar un nuevo SIS se necesita [3]:

- Contar con marcos regulatorios.

- Un buen nivel de estandarización.

- Compatibilidad entre los sistemas.

- Una buena estrategia de formación de recursos humanos.

- Infraestructura tecnológica.

- Selección de equipos de trabajo multidisciplinario.

- Personal capacitado en sistemas de información en salud.

Antes de desarrollar un nuevo sistema de información en salud, se debe contar con los objetivos del sistema nacional de salud, los cuales se alcanzan abriendo diálogos y espacios de discusión entre gobierno, universidades y sistemas de provisión de salud. Como resultado de los diálogos se deberá tener la definición del sistema de salud, su estructura, objetivos nacionales y definir prioridades. La incorporación de las TIC a los sistemas de salud ayuda a cumplir con los objetivos y además brinda las facilidades para el acceso a las personas a una atención oportuna y de calidad [4].

Gasto en Salud Ecuador (\%PIB)

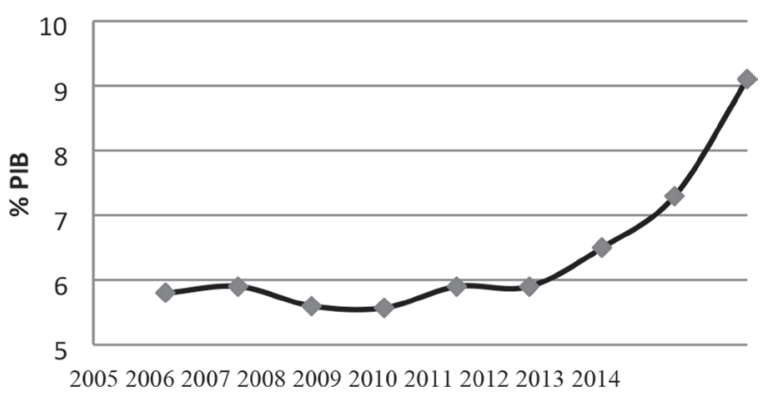

Figura 1. Inversión en salud con respecto al PIB. Fuente: Banco Mundial. 
Hay países con sistemas universales de salud en donde el paciente es el beneficiario, al contar con mejores servicios de salud y sin tener que pagar altos costos por su atención. En estos países los gobiernos son los encargados de regular, facilitar o proveer los componentes de un SIS, a la vez que son los llamados a cumplir las siguientes actividades para la planificación y gestión de los recursos del sistema:

- Definición y reglamentación de leyes.

- Regulación del sector de la salud.

- Financiamiento del sistema con recursos públicos

- La contratación de servicios en redes públicas y/o público-privadas.

- Provisión directa de servicios a través de redes públicas y universitarias.

Los países de la región y del mundo, en la mayoría de los casos han utilizado las estrategias topdown, bottom-up y middle-out, para la planificación de los sistemas de información en salud [5]. En la primera estrategia los gobiernos imponen para toda la nación el sistema de información que los profesionales de la salud y las instituciones deben usar. En la estrategia bottom-up, en cambio, cada institución de salud decide en forma libre y autónoma el sistema a utilizar, siempre y cuando cumpla con la reglamentación y leyes de cada país. Finalmente, la tercera estrategia es la que más se ajusta a las necesidades de los profesionales de la salud, la industria de las TIC y el gobierno; en esta estrategia se deben llegar a acuerdos para el desarrollo de objetivos comunes, desarrollo de estándares, y soporte a las implementaciones que beneficien a todos, el gobierno toma un papel de liderazgo como sucedió en Australia que dirigió sus inversiones al establecimiento de normas a escala nacional, mucho antes de contemplar el desarrollo o la compra de cualquier sistema. Esta estrategia enriquece la capacidad para compartir información y brindar datos de mayor calidad.

Una manera eficiente de construir sistemas informáticos para el área de la salud, es mediante la utilización de estándares y herramientas de software que permitan intercambiar información entre los diferentes sistemas, así como se realizó en la implementación del proyecto EHRG [6] en Uruguay. Una herramienta de software para la creación de sistemas de HCE que buscó solucionar la mayoría de problemas y riesgos pre- sentados en la construcción y mantenimiento de software para el área clínica. El uso de estos estándares y herramientas lograron que exista una mejor comunicación entre los profesionales de la salud e informáticos, mayor rapidez en la implementación de cambios, mantenimiento de la HCE a largo plazo y reducir los costos en tiempo y recursos humanos.

En Suecia, los ciudadanos cuentan con acceso universal a los servicios de salud, y las personas pueden acudir a las instituciones de salud públicas o privadas, sin importar sus ingresos. En cambio en Francia los habitantes pueden ir al médico privado, y luego la Seguridad Social reembolsa el valor de la consulta. En España solo los funcionarios públicos pueden escoger entre un servicio de salud privado o público [7].

En la Región de las Américas, existen varias estrategias y planes de acción, con el fin de lograr que más sectores de la población de los países miembros, tengan acceso a servicios de salud de calidad, con la ayuda de las tecnologías de la información y la comunicación. El acceso a las TIC no es universal, y muchos países y poblaciones de la región disponen de manera desigual de estas herramientas [3]. El propósito es contribuir al desarrollo sostenible de los sistemas de salud de sus estados miembros y con su adopción se busca mejorar el acceso a los servicios de salud y su calidad, gracias a la utilización de las tecnologías de la información y comunicación. La implementación está apoyada por la Organización Panamericana de la salud (OPS) y la Organización Mundial de la Salud (OMS), quienes a través de normativas regionales y nacionales impulsan para que los países de la región cuenten con registros médicos electrónicos, servicios de telesalud, mSalud (dispositivos móviles) y educación en TIC, para disminuir la brecha tecnológica bajo el concepto de "alfabetización digital" [8].

Si bien todos los países, sin excepción, tienen grandes avances en la implementación de tecnologías de información en salud, los países con los que la OPS ha venido avanzando en estrategias nacionales de eSalud son: Argentina, Brasil, Chile, Costa Rica, República Dominicana, Guatemala, Jamaica, México, Panamá, Paraguay, Perú y Trinidad y Tobago.

En diciembre de 2013, la empresa Health Digital Systems (HDS) comenzó el trabajo de dimensionamiento para el proyecto "Solución 
Informática para la Gestión Integral en Salud" (SIGIS) impulsado por el Ministerio de Salud Pública del Ecuador, que tiene como objetivo fundamental contar con la capacidad de administrar el Sistema de Salud de Ecuador en su totalidad, a nivel clínico, administrativo, financiero y de regulación. El alcance de esta propuesta incluye análisis de información en salud, apoyo a la atención médica a través del Expediente Clínico Electrónico y Administración de la Red de Servicios. El contrato incluye la suite "Dendritas" diseñada específicamente para Redes Integradas de Salud, lo que implica la implantación del Expediente Médico Administrativo (EMA).

Desde el año 2013 se implementó el proyecto "SIGIS" en 116 instituciones de salud. Este sistema facilita a los médicos ecuatorianos obtener datos en tiempo real (historias clínicas a nivel nacional) con el fin de mejorar la calidad en la atención y tomar decisiones basadas en un monitoreo de resultados sanitarios [9]. Se espera que hasta finales del 2016 se implemente en 151 centros, distribuidos a nivel nacional.

Como parte de la instalación del sistema, se ha equipado con infraestructura informática las distintas unidades de salud, con el fin de lograr que todos los funcionarios cuenten con una computadora y tengan acceso al nuevo sistema. También se ha equipado a los hospitales y centros de salud con equipos para conectividad $\mathrm{WiFi}$, dotación de internet, puntos de red, cableado estructurado, lo cual ha facilitado el acceso a los registros médicos de los pacientes desde cualquier lugar y a cualquier hora [10].

La empresa pública Yachay firmó un acuerdo de entendimiento con la empresa mexicana Health Digital Systems, para implementar una casa de software y un centro de investigación y desarrollo en la ciudad del conocimiento Yachay. Esta casa de generación de conocimiento y de la industria del software pondrá énfasis en los sistemas de administración de la salud, para mejorar las condiciones de vida de los ciudadanos.

El presupuesto del Ministerio de Salud Pública (MSP) con respecto al Presupuesto General del Estado (PGE), se ha incrementado como se presenta en la figura 2. Si bien la ley proclama la cobertura universal de salud, en los hechos existe una limitada cobertura real con servicios cuya calidad no es la óptima.

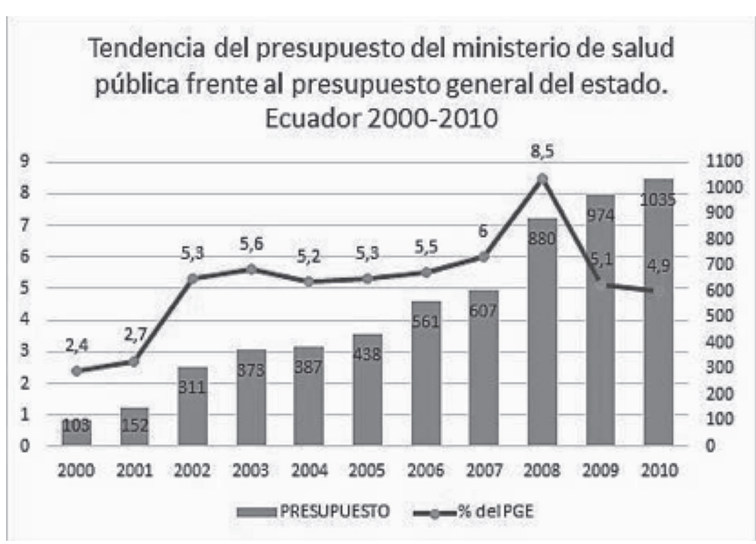

Figura 2. Tendencia del presupuesto para el Ministerio de Salud Pública con respecto al PGE.

Fuente: MSP. Presupuesto del Estado, años 2000-2010.

La cobertura de salud de las instituciones públicas y privadas cubre aproximadamente al 79\% de la población. Así, el IESS (Instituto Ecuatoriano de Seguridad Social) atiende al $20 \%$ de la población, el ISSFA (Instituto de Seguridad Social de las Fuerzas Armadas del Ecuador) y el ISSPOL (Instituto de Seguridad Social de la Policía Nacional) cubren a poco más del $5 \%$ de la población. El MSP a alrededor del 35\% de la población ecuatoriana. Los seguros privados y empresas de medicina prepagada al $10 \%$ de la población, perteneciente a estratos de ingresos medios y altos; la Junta de Beneficencia y SOLCA (Sociedad de Lucha Contra el Cáncer del Ecuador) atienden a alrededor del 5\% de la población. En la figura 3, se muestran estas cifras, que representan la cobertura de los servicios de salud en el Ecuador. Las instituciones con mayor cobertura son el MSP y el IESS, ya que entre las dos dan cobertura a cerca del 55\% de la población ecuatoriana.

\section{Componentes para la planificación estratégica de un sistema de información en salud}

Los componentes dependen de cada sistema de información y la estrategia se basa en los objetivos del sistema de salud de cada país. Los países que han tenido éxito en el desarrollo de sistemas de información en salud, han utilizado los siguientes componentes: gobierno y gestión de los servicios de las TIC; planificación y gestión de proyectos; adecuado manejo del cambio; recurso humano especializado; manejo de infraestructura; interoperabilidad y estándares claros; registro médico electrónico; servicios terminológicos; se- 
guridad de la información en salud y soporte para la toma de decisiones [3].

Existen experiencias exitosas de creación de sistemas de información en salud en la región. Entre los principales se tiene: Sistema de Información de la Red Asistencial (SIDRA) en Chile; Proyecto Itálica del Hospital Italiano de Buenos Aires Argentina; la Agencia AGESIC en Uruguay y la Propuesta de un conjunto de estándares y el marco legal necesario en Brasil.

\section{Historia Clínica Electrónica}

La historia clínica es un documento que cada vez que un paciente acude a una institución de salud, pública o privada, debe ser llenado por un profesional de la salud. Su información básica consta de los datos personales y clínicos del paciente. Disponer de registros médicos en forma electrónica puede facilitar el acceso a la información, la comunicación y la adopción de medidas que ayuden a mejorar la calidad de la salud y la seguridad de los pacientes [11]. Impulsar el uso del "Registro Electrónico de Salud" (RES) y capacitar e informar a toda la población sobre la importancia del cuidado apropiado de la información clínica es imperativo. La OPS fomenta el desarrollo de sistemas locales, a través de la capacitación y equipamiento a gobiernos y organizaciones no gubernamentales.

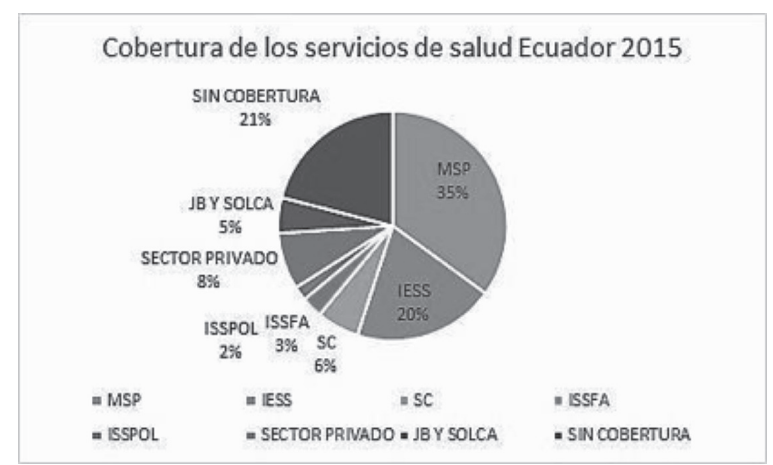

Figura 3. Cobertura de los sistemas de salud en Ecuador.

Fuente: Sistema de salud de Ecuador, 2015.

Contar con políticas nacionales claras sobre estándares de salud es un primer paso que deben dar los gobiernos, antes de implementar cualquier sistema de información en salud. Con el establecimiento de políticas y normas nacionales se logrará intercambiar información entre insti- tuciones para obtener el máximo beneficio de los registros. Los registros electrónicos son la puerta principal de entrada de datos al sistema de información por parte de los profesionales de la salud. La historia clínica, está formada por documentos, tanto escritos como gráficos que contienen información de salud y enfermedades de una persona.

En el Ecuador y en muchos países, la historia clínica todavía se maneja en papel por los profesionales de la salud, con todas las limitaciones y riesgos que esto trae. En los últimos años se ha promovido el uso del RES como una manera de disminuir los riesgos y desventajas del uso del papel.

Existen muchas ventajas de utilizar las historias clínicas electrónicas, entre las más relevantes se tiene: la disponibilidad, la legibilidad, el almacenamiento, la calidad de la información, la durabilidad y la seguridad de la información almacenada. Hay muchos términos que se utilizan para referirse a la historia clínica electrónica, tales como: registro electrónico de salud, registro médico computarizado, ficha clínica electrónica. Así como existen muchos términos también hay varias definiciones, pero la que ha sido acogida por la gran mayoría es la propuesta por la IEEE que la define como: "Registro de datos de salud propios de un sujeto humano, en formato digital y que incluye el estado de la salud en distintos momentos de la vida y las acciones que se desarrollan para determinarlo y/o modificarlo".

También los RES permiten la integración con otros componentes del sistema de información en salud, como resultados de laboratorio o de imágenes. Los RES tienen muchas funcionalidades de acuerdo a un informe del Instituto de Medicina de los Estados Unidos (IOM), siendo las áreas funcionales claves las siguientes:

- Gestión de la información.

- Manejo de resultados.

- Manejo de órdenes médicas.

- Sistemas que ayudan a una mejor toma de decisiones.

- Ayuda o soporte a los pacientes.

- Integración con sistemas administrativos.

- Posibilidad de generar informes de salud pública.

El estándar ISO 18308 sobre RES [12], también lista una serie de requerimientos de arquitectura en línea con las áreas funcionales descritas por el IOM: 
- La inclusión de información en texto libre.

- La búsqueda en datos no estructurados (textual y no textual).

- La inclusión de texto estructurado dentro de esos datos.

- Posibilidad de inclusión de comentarios dentro de la información almacenada, lo que permite al médico calificar la información adecuadamente.

Actualmente una persona puede tener muchas historias clínicas, dependiendo del número de establecimientos sanitarios a los cuales ha acudido. Si un paciente se acerca a un hospital y no tiene abierto una historia clínica o su historia se ha extraviado, el médico abre una nueva historia clínica. Una persona puede llegar a tener varias historias clínicas dependiendo del número de establecimientos de salud visitados a lo largo de toda su vida.

La historia clínica electrónica, elimina la repetición de historias de un mismo paciente. Según la International Organization for Standardization (ISO), una HCE es "un repositorio de datos de un paciente en un formato digital, almacenados de una manera segura y accesible a usuarios autorizados" [13]. Una HCE almacena y organiza la información del paciente de una forma diferente y gracias a las TIC permite acceder a la información desde cualquier lugar, en cualquier momento, por parte de los profesionales de la salud. En el Ecuador ya se cuenta con HCE, en establecimientos de salud privados y en varios hospitales y centros de salud pertenecientes al Ministerio de Salud Pública.

La estructura de las HCE ha variado con el tiempo, anteriormente se utilizaba HCE orientadas al tiempo, orientadas a los problemas y orientadas a la fuente de información. Hoy en día las HCE combinan los tres elementos [14].

Las historias clínicas digitales se implementaron desde enero de 2016 en el Hospital "San Vicente de Paúl" de la ciudad de Ibarra, y como parte de esta implementación los profesionales de la salud están recibiendo cursos de capacitación dictados por funcionarios del Health Digital System (HDS), representante del sistema "Si Salud" en el Ecuador. La inversión contratada con la empresa para la implementación de este sistema de información en salud a nivel nacional asciende a USD 19.700.000. A este valor hay que añadir la compra de equipos y herramientas de comunicación tecnológica [15].

\section{E. Situación actual y perspectivas de la historia clínica electrónica}

En Europa se obtuvieron buenos resultados al utilizar las HCE. En la comunidad de Madrid se utilizó las historias clínicas electrónicas para la vigilancia de la gripe pandémica (H1N1) durante el 2009 y el 2010, al contar con información muy útil para el seguimiento de la evolución de la pandemia y la toma de decisiones a tiempo [16].

La evaluación y certificación de los RES es un problema debido a que no existe reglamentación clara en los países, excepto en Brasil. En algunos países existen entes certificadores, que deben ser aplicados en los sistemas de salud para poder contar con la certificación del software utilizado.

A pesar de las ventajas claramente documentadas en varios países, se presentan algunos riesgos o problemas que pueden ser evitados al monitorear los procesos de implementación de los RES.

Existen experiencias exitosas de implementación de los RES en varios países de la región:

- Proyecto Itálica del Hospital Italiano de Buenos Aires (HIBA) en Argentina.

- Federación Médica del Interior (FEMI) en Uruguay.

- Megasalud en Chile.

- Clínica Alemana en Chile.

- Fundación Cardioinfantil Instituto de Cardiología en Colombia.

\section{F. Estándares para la Interoperabilidad}

La falta de acuerdos sobre estándares, evita el intercambio de información entre los sistemas electrónicos y administrativos que posee una institución de salud. Acuerdos en modelos de información, protocolos de comunicación y formatos de intercambio de datos, se requiere para tener sistemas de información interoperables dentro de un sistema de salud, para lograr mejoras en la calidad asistencial.

Es necesario llegar a acuerdos regionales y nacionales para el desarrollo de estándares, sin descuidar los objetivos del sistema de salud. Actualmente varias organizaciones de salud poseen diferentes tipos de registros electrónicos, ya sea clínicos o administrativos, en donde los datos clínicos del paciente son el corazón del sistema de información. Es importante tener estándares que faciliten la interoperabilidad. 
La interoperabilidad es la "habilidad o capacidad de dos o más sistemas para intercambiar información y utilizarla".

Es recomendable implementar estándares como:

- Health Level Seven (HL7)

- Digital Imaging on Communication in Medicine (DICOM)

- Organización Internacional de Normalización 13606 (ISO 13606)

- Continuity of Care Record (CCR)

- OpenEHR.

\section{G. Tipos de interoperabilidad}

Existen diferentes niveles de interoperabilidad [17]. En primer lugar, desde el punto de vista de la lógica de sistemas se cuenta con la interoperabilidad sintáctica, la interoperabilidad semántica y la interoperabilidad de negocios u organizativa. Desde el punto de vista de la arquitectura de sistemas se puede clasificar en nivel 1 , en el cual cada sistema individual debe cumplir con un nivel básico de estandarización; el nivel 2 tiene que ver con las redes y la aplicación de estándares como protocolos de comunicación e interfaces, y finalmente el nivel 3 se encarga de la infraestructura de información y servicios para la interconexión de redes.

\section{H. Categorías de estándares de Interoperabilidad}

Dependiendo del tipo de información que se quiera intercambiar existen diferentes categorías de estándares que han sido utilizados en varios países, para lograr la interoperabilidad de los sistemas. Para el intercambio de datos y mensajería se utilizan los estándares: HL7, DICOM e IEEE. Para el manejo de la terminología se cuenta con: Systematized Nomenclatura of Medicine (SNOMED), para términos clínicos; Logical Observation Identifiers Names and Codes (LOINC), para resultados de laboratorio; Clasificación Internacional de Enfermedades (CIE) para enfermedades y causas de muerte; Clasificación Internacional de Atención Primaria (CIAP). Para manejo de documentos se tiene: Clinical Document Architecture (CDA); American Section of the International Association for Testing Materials International (ASTM); Continuity of Care Record (CCR); y CCD estándar de documentos creados en colaboración entre HL7 y ASTM.
Para el caso de las aplicaciones se puede mencionar: Clinical Context Object Workgroup (CCOW), para el login único para varias aplicaciones. A nivel conceptual se tiene los siguientes estándares: Modelo de Información de Referencia (RIM); Modelo Funcional del Instituto of Medicine (IOM); Modelo Funcional HL7 y Modelo de OpenEHR. Y finalmente en la categoría de arquitectura se han desarrollado los siguientes estándares: Centro para el Control y la Prevención de Enfermedades (CDC) y el Sistema de Registro Electrónico Nacional de Enfermedades.

Dentro de las barreras para lograr la interoperabilidad se encuentran la complejidad técnica y los aspectos políticos que dificultan el desarrollo tecnológico.

\section{Resultados y discusión}

Las estrategias utilizadas para el desarrollo de los sistemas de información en salud, en los países de la región de las Américas no son las adecuadas, ya que por una parte el sector público implementa sistemas de salud para sus centros y hospitales a través de la contratación pública, con empresas desarrolladoras de software clínico y con recursos del Presupuesto General del Estado, mientras que las empresas privadas, entre ellas los consultorios médicos, las clínicas privadas y los hospitales de este sector, desarrollan sistemas informatizados de salud con sus propios recursos y sin un mayor acatamiento de las normas y reglamentos existentes a nivel país.

Al final se tiene dos sectores aislados y con sus propios sistemas de información en salud, que serán muy difíciles de integrar en una única solución informática para la gestión integral en salud. No usar estándares para la interoperabilidad de los sistemas, es el principal error cometido por los países de la región, en el desarrollo de los sistemas de registros médicos electrónicos. Una solución para la integración de los sistemas de información en salud de las instituciones públicas y privadas, sería a través del uso de servicios web basados en XML, a través de una arquitectura orientada a servicios (SOA), para lo cual sería conveniente que cada institución en primer lugar comience a crear ambientes SOA.

\section{Conclusiones}

Los sistemas informáticos de salud mejoran los servicios de salud de los ciudadanos, al contar 
con herramientas para que los profesionales de la salud puedan acceder a la información clínica y personal de los pacientes desde cualquier lugar, a cualquier hora y con la facilidad de acceso desde múltiples dispositivos electrónicos.

Las historias clínicas electrónicas tienen grandes beneficios si se las compara con las historias clínicas en papel, principalmente en el acceso, la disponibilidad de la información, la legibilidad, la seguridad y la integración con otros componentes del sistema de información. Países que al desarrollar sistemas de información en salud identificaron en primer lugar los objetivos del sistema nacional de salud, llegaron a acuerdos en cuanto a estánda- res, formatos, protocolos de comunicación, tuvieron gran éxito y en la actualidad cuentan con un sistema universal de salud, que brinda atención de calidad y de manera oportuna a todos los habitantes, sin importar sus ingresos económicos.

El desarrollo de sistemas de información en salud utilizando servicios web basados en XML y a través de una arquitectura orientada a servicios a menudo es una solución prometedora para los problemas de integración, permitiendo reutilizar aplicaciones existentes y usando una tecnología fundamental para dominar y manejar la complejidad y heterogeneidad de los sistemas de información en salud.

\section{Bibliografía}

[1] Luna, D., Soriano, E., \& de Quirós, F. G. B. (2007). Historia clínica electrónica. Revista del Hospital Italiano de Buenos Aires, 27(2).

[2] Laudon C., Laudon P. (2012). Creación de sistemas de información. Sistemas de información gerencial (pp. 489-490). México: Pearson

[3] Organización Panamericana de la Salud. (2014). Conversaciones sobre eSalud: Gestión de información, diálogos e intercambio de conocimientos para acercarnos al acceso universal a la salud.

[4] Carnicero, J., \& Fernández, A. (2012). Manual de salud electrónica para directivos de servicios y sistemas de salud.

[5] Coiera, E. (2009). Building a national health IT system from the middle out. Journal of the American Medical Informatics Association, 16(3), 271-273.

[6] Pazos, P. (2012). Ehrgen: Generador de sistemas normalizados de historia clínica electrónica basados en openehr. En 3er Congreso Argentino de Informática y Salud.

[7] P. I. Juárez (2015, febrero). "Sanidad de acceso universal, pública o privada: En Suecia lo hicieron".

[8] D Agostino, M. (2015). Estrategias de salud electrónica en la región de las Américas: situación actual y perspectivas. Revista Peruana de Medicina Experimental y Salud Pública, 32(2), 352-355.

[9] C. A. Corral, (2016, enero). "Proyecto SI salud se implementara en el HDTC".

[10] K. Morejón, (2014, noviembre). "Proyecto Si Salud se implementa en el norte del país”, disponible en http://www.elciudadano.gob.ec/proyecto-si-salud-seimplementa-en-el-norte-del-pais/

[11] Otero, P. (2011). Beneficios y riesgos relacionados con el uso de la historia clínica electrónica. Archivos argentinos de pediatría, 109(6), 476-477.

[12] ISO 18308: 2011 - Health informatics - Requirements for an electronic health record architecture [Internet]. Disponible en: http://www.iso.org/iso/catalogue detail. htm? csnumber=52823

[13] Rojas Mezarina, L., Medina, C., Alicia, C., \& Vargas Herrera, J. (2015). Registro nacional de historias clínicas electrónicas en Perú. Revista Peruana de Medicina Experimental y Salud Publica, 32(2), 395-396.

[14] ISO/DTR 20514, (2004) Health Informatics - Electronic Health Record - Definition, Scope, and Context.

[15] M. Rodríguez, (2015, noviembre). "Nueva era digital para hospital San Vicente de Paúl", disponible en http://hsvp.gob.ec/index.php/component/k2/item/72-nuevaera-digital-para-hospital-san-vicente-de-paul 
[16] Esteban-Vasallo, M. D., Domínguez-Berjón, M., Génova Maleras, R., Blanco Ancos, L. M., Astray Mochales, J., Lópaz Pérez, M., \& Aragon Peña, A. (2010). Vigilancia diaria de la gripe pandémica (H1N1) 2009 mediante registros de la historia clínica electrónica de atención primaria en la comunidad de Madrid. Revista española de salud pública, 84(5), 657663.

[17] Indarte, S., \& Pazos Gutiérrez, P. (2011). Estándares e interoperabilidad en salud electrónica: requisitos para una gestión sanitaria efectiva y eficiente.

[18] Barker, A., Manji, F., Charlton, J., Escribir para el cambio, disponible en: http://www.fahamu. org/WFCSpanish/sitemap.html

[19] Perú, Congreso de la República. Ley N 1243. Ley que crea el Registro Nacional de Historias Clínicas Electrónicas.

Lima: Congreso de la República, 2013. 